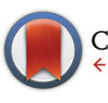

CrossMark $\leftarrow$ click for updates

Cite this: Dalton Trans., 2015, 44 19509

Received 24th June 2015,

Accepted 16th July 2015

DOI: $10.1039 / c 5 d t 02398 j$

www.rsc.org/dalton

\section{Axial fluoride binding by lanthanide DTMA complexes alters the local crystal field, resulting in dramatic spectroscopic changes $\uparrow$}

\author{
Octavia A. Blackburn, ${ }^{a}$ Alan M. Kenwright, ${ }^{\text {b }}$ Paul D. Beer ${ }^{a}$ and Stephen Faulkner*a \\ Addition of fluoride to aqueous solutions of lanthanide complexes of DTMA results in the formation of \\ ternary complexes of the form [F.Ln.DTMA $]^{2+}$ in which an axial solvent molecule is displaced by fluoride. \\ $[\mathrm{F} \cdot \mathrm{Ln} \cdot \mathrm{DTMA}]^{2+}$ and $\left[\mathrm{H}_{2} \mathrm{O} \cdot \mathrm{Ln} \cdot \mathrm{DTMA}\right]^{3+}$ are in exchange on a timescale of around $1 \mathrm{~s}$. Dramatic changes are \\ observed in both the NMR and luminescence spectra of the complexes: these are consistent with a change \\ in the nature of the magnetic anisotropy at the paramagnetic lanthanide centre, itself arising from a change \\ in the local crystal field. Study of paramagnetic lanthanide complexes with anisotropic electronic distri- \\ butions reveals that, upon replacing water with fluoride, there is an inversion of the sign, and a significant \\ reduction in the magnitude, of the crystal field term that defines the nature of the pseudocontact shift.
}

The spectroscopic properties of lanthanide complexes have been studied in depth for many years as a consequence of their utility in a wide variety of applications. For the last three decades, the application of such complexes has been dominated by the use of gadolinium complexes in magnetic resonance imaging (MRI) and the use of luminescent lanthanide complexes in time-resolved bioassays. ${ }^{1}$ More recently, magnetic resonance spectroscopy methods for imaging have been diversified to other lanthanide complexes through the exploration of PARACEST and PARASHIFT probes, ${ }^{2}$ while the molecular magnetism of paramagnetic lanthanide complexes has become a burgeoning field of interest. ${ }^{3}$ Furthermore, the use of polydentate ligands that incompletely saturate the lanthanide coordination sphere has resulted in a diverse range of systems in which solvent is displaced by anionic analytes, giving responsive systems that can be used to probe analyte concentration, or applied in early diagnosis of disease. ${ }^{4}$

All of these studies are only possible because of the fundamental properties of paramagnetic lanthanide complexes. Among the tripositive ions, gadolinium is exceptional, in that its $4 \mathrm{f}^{7}$ configuration is defined by a ${ }^{8} \mathrm{~S}_{7 / 2}$ ground state with a spherical electronic distribution in the free ion. For all the other paramagnetic lanthanide(III) ions, there is inherent an-

\footnotetext{
${ }^{a}$ University of Oxford, Chemistry Research Laboratory, 12 Mansfield Road, Oxford OX1 3TA, UK. E-mail: Stephen.Faulkner@chem.ox.ac.uk

${ }^{b}$ Department of Chemistry, University of Durham, South Road, Durham DH1 3LE, UK. E-mail: a.m.kenwright@dur.ac.uk

$\dagger$ Electronic supplementary information (ESI) available: NMR experimental detail; titration procedures, isotherms and data fitting; luminescence spectra and decays; ${ }^{1} \mathrm{H}$ and ${ }^{19} \mathrm{~F}$ NMR spectra; Bleaney and Reilley plots and corresponding data. See DOI: 10.1039/c5dt02398j
}

isotropy to the electronic distribution around the metal centre. Although crystal field effects for lanthanide complexes are small by comparison with the d-block transition metals, the crystal field still has significant consequences for the behaviour of lanthanide-containing systems.

For more than forty years, Bleaney's ideas have dominated our understanding of the NMR of lanthanide complexes. ${ }^{5}$ Bleaney pointed out that the observed chemical shift for a given nucleus can be defined in terms of the sum of dipolar or pseudocontact (through-space) and contact (through-bond) contributions, in addition to the usual diamagnetic shift. In most lanthanide-containing complexes, the pseudocontact term, $\delta_{\mathrm{P}}$, is the dominant factor in determining the observed shift for the nuclei of common ligand architectures. For an axially symmetric system,

$$
\delta_{\mathrm{P}}=\frac{2 C_{J} \beta^{2}}{(k T)^{2}} \frac{\left(3 \cos ^{2} \theta-1\right) B_{0}^{2}}{r^{3}}
$$

where $\theta$ and $r$ are the polar coordinates relative to the principal axis with the lanthanide at the origin, $B_{0}^{2}$ is the second order crystal field parameter, and $C_{J}=g^{2} J(J+1)(2 J-1)(2 J+3)\left\langle J|a| J^{\prime}\right\rangle$. Clearly, the sign and magnitude of this effect depend upon the lanthanide under study, and the lanthanide ions have traditionally been divided into those with prolate electronic distributions (i.e. with the spherical distortion resulting in an extension along the molecular axis), and those with oblate electronic distributions (i.e. with the distortion aligned perpendicular to the molecular axis).

Crystal field effects also influence the photophysical properties of the lanthanides, though to a less profound degree. The fine structure of the observed transitions in optical 
spectra is a direct consequence of crystal field effects: in luminescence spectra, this is a consequence of the degeneracies of both ground and excited states being lifted by the ligand field.

Despite the rapid development of the field in general, there has been relatively little work done to correlate local ligand field effects with the properties of complexes. Parker and coworkers explored the effect of changing solvent (and thus axial donor) on lanthanide tetraamide complexes, ${ }^{6}$ and have recently studied the scope and limitations of Bleaney's theory applied to a range of complexes. ${ }^{7}$

It has now become apparent that the crystal field splitting (including higher order terms) plays a more significant part in determining the observed properties of lanthanide complexes (including the electronic relaxation time, $\left.T_{1 \mathrm{e}}\right)^{8}$ than was previously supposed, and that the ability to manipulate and control this parameter is likely to play an important role in the design of new complexes. The observation of dramatic changes in this parameter on ligand binding is therefore of significance.

Study of the interactions of halide guests with stable octadentate complexes are equally rare. Aime et al. observed that addition of fluoride caused significant changes to the NMR spectra of lanthanide-DOTA complexes. ${ }^{9}$ More recently, Charbonnière and co-workers have observed strong fluoride binding in $C_{2}$ symmetric systems, though without discussing the consequences of binding on ligand field. ${ }^{10}$

This manuscript presents a detailed study of the interactions of paramagnetic lanthanide complexes with fluoride ions in water, using luminescence and NMR spectroscopies to characterise the effect of the changes in ligand field on the observed spectra. This work extends our recent work on the ytterbium complex of DTMA to the whole series. ${ }^{11}$

\section{Materials and methods}

The tetraamide ligand DTMA (1,4,7,10-tetrakis[( $N$-methylcarbamoyl)methyl]-1,4,7,10-tetraaza-cyclododecane) and its lanthanide complexes ( $\mathrm{Pr}$ to $\mathrm{Lu}$, minus Gd with $\mathrm{Pm}$, plus $\mathrm{Y}$ ) were synthesised as their trifluoromethanesulfonate salts, as previously described. ${ }^{12}$ Full details of methods used for NMR and luminescence spectroscopies and data fitting can be found in the ESI $\dagger$ associated with this paper.

\section{Results and discussion}

\section{Luminescence spectroscopy}

The emission spectrum of EuDTMA was recorded in the presence and absence of sodium fluoride in aqueous solution (Fig. 1). Significant changes are observed in both the form and intensity of the bands, in particular the $\Delta J=1$ and $\Delta J=4$ bands show a marked change in shape. The nature of the Stark splitting of the $\Delta J=1$ band $(c a .590 \mathrm{~nm})$ has been used as a direct measure of the magnitude, and sometimes sign, of the second order crystal field coefficient, $B_{0}^{2}$, in axially sym-
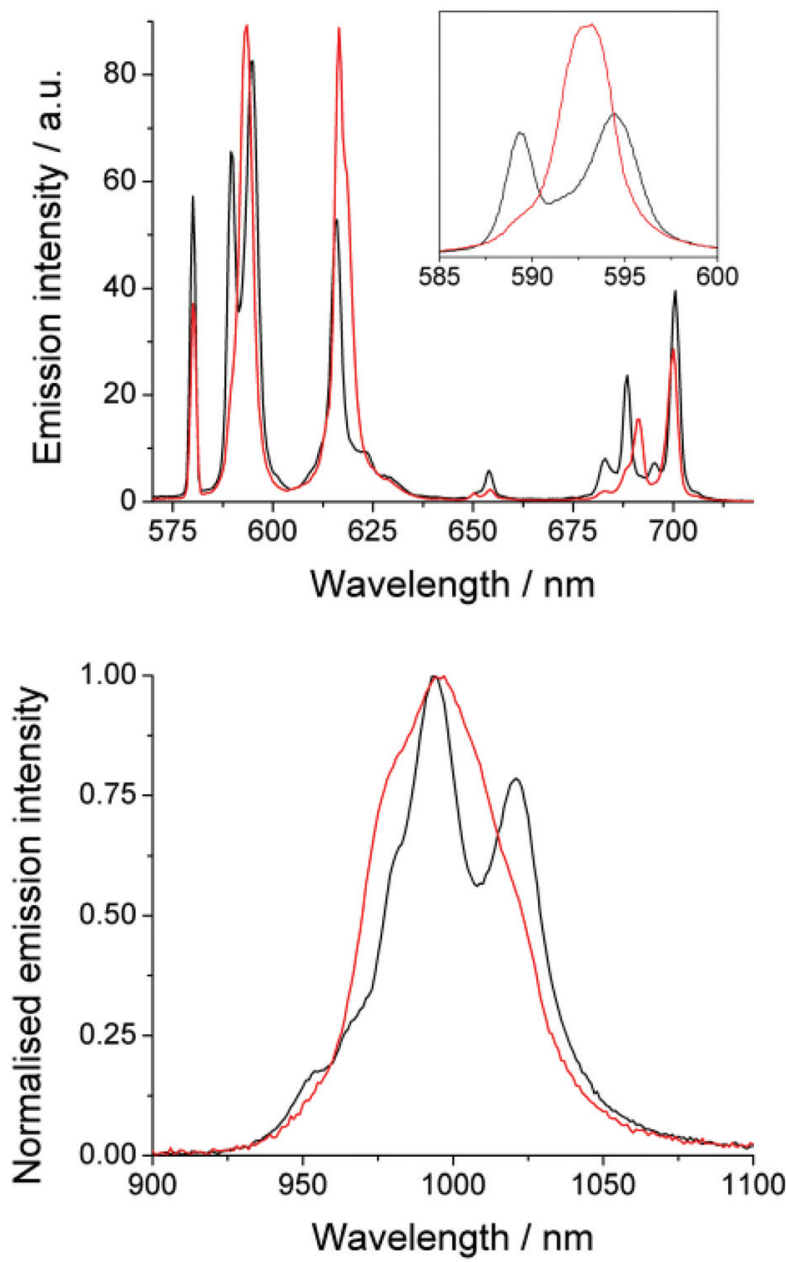

Fig. 1 Change in form of emission spectra with fluoride. Top: EuDTMA in $\mathrm{D}_{2} \mathrm{O}$ (black) and with excess sodium fluoride (red), $\lambda_{\mathrm{ex}}=394 \mathrm{~nm}$, exit slit = $1 \mathrm{~nm}$; inset shows the change in structure of the $\Delta J=1$ band measured with $0.2 \mathrm{~nm}$ exit slit. Bottom: YbDTMA in $\mathrm{D}_{2} \mathrm{O}$ (black) and with added fluoride (red), $\lambda_{\mathrm{ex}}=265 \mathrm{~nm}$, exit slit $=5 \mathrm{~nm}$.

metric systems. ${ }^{13}$ Upon addition of fluoride, the Stark splitting of $\Delta J=1$ dramatically decreases and $\mathrm{A}$ and $\mathrm{E}$ components merge to form one apparent peak. This indicates that the second order crystal field parameter has dramatically decreased in magnitude. Information about any change in sign of $B_{0}^{2}$ is unresolvable, even at narrower slit widths. Such a striking effect on $B_{0}^{2}$ would indicate a significant change to the crystal field around the europium centre.

Near-IR emission from YbDTMA was also recorded in the presence and absence of fluoride upon excitation at $265 \mathrm{~nm}$ (Fig. 1). Once again, the shape of the spectrum is significantly altered. Although interpretation of the Stark splitting is much more complex due to the high $J$-multiplicity of the excited $\left({ }^{2} \mathrm{~F}_{5 / 2}\right)$ and ground $\left({ }^{2} \mathrm{~F}_{7 / 2}\right)$ states, we can infer a change in $B_{0}^{2}$ analogous to that observed with Eu.

The effects of fluoride on the luminescence lifetimes of EuDTMA were also investigated. The Eu-based lifetime increased on addition of excess fluoride from 0.54 to $1.07 \mathrm{~ms}$ 


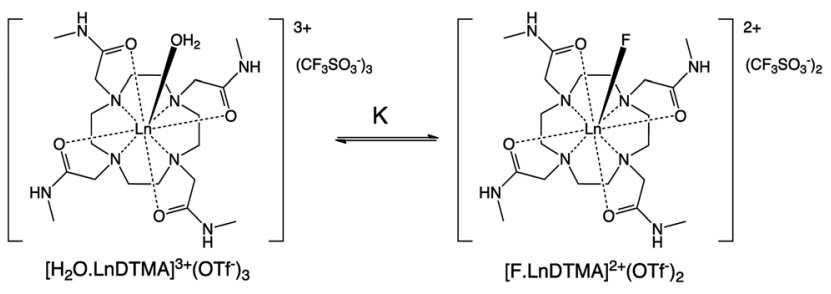

Scheme 1 The equilibrium between hydrated and fluoride-bound LnDTMA complexes under study.

in $\mathrm{H}_{2} \mathrm{O}$, and 1.72 to $2.41 \mathrm{~ms}$ in $\mathrm{D}_{2} \mathrm{O}$. This corresponds to a change in the number of bound water molecules, $q,{ }^{14}$ from 0.9 to 0 , indicating that the axially bound water is displaced by fluoride (Scheme 1).

\section{NMR studies on diamagnetic complexes}

The effect of fluoride on the proton NMR spectra of LuDTMA and YDTMA complexes was measured in $\mathrm{D}_{2} \mathrm{O}$ (see ESI $\dagger$ ). Addition of sodium fluoride to a solution of LuDTMA caused a significant shifting of the resonances corresponding to the $\mathrm{CH}_{2}$ cyclen ring protons and the amide arm protons, in line with a fast exchange process on this timescale. In the case of YDTMA, the exchange appears to be slower, with a new set of resonances emerging while the original peaks diminish, although some shifting of the peaks indicates an intermediate exchange rate (i.e. the exchange rate is non-negligible on the relevant NMR timescales).

The ${ }^{19} \mathrm{~F}$ NMR proves to be more revealing. In addition to the expected fluoride peak at $-122 \mathrm{ppm}$, resonances are also found at -74 and -58 ppm for LuDTMA and YDTMA respectively (see $\mathrm{ESI}_{\dagger} \dagger$ ). Saturation transfer experiments reveal that these peaks are in exchange with those corresponding to free fluoride at $-122 \mathrm{ppm}$. The $-74 \mathrm{ppm}$ peak in the LuDTMA spectrum is broader than that at $-58 \mathrm{ppm}$ with YDTMA at $298 \mathrm{~K}$, but sharpens up on cooling in corroboration with the faster exchange rate observed in the ${ }^{1} \mathrm{H}$ NMR. More interestingly, the peak at $-58 \mathrm{ppm}$ in the YDTMA spectrum is a doublet with a coupling constant of $64 \mathrm{~Hz}$, which remains when the spectrum is recorded with proton decoupling and does not vary with the applied field. This splitting is indicative of traditional spin-spin coupling between the ${ }^{19} \mathrm{~F}$ and ${ }^{89} \mathrm{Y}$ nuclei. The presence of ${ }^{89} \mathrm{Y}-{ }^{19} \mathrm{~F} J$-coupling was confirmed by ${ }^{19} \mathrm{~F}$ detected HMQC (Fig. 2). These observations indicate that fluoride is bound at the lanthanide centre and is in exchange with bulk fluoride in a process that is slow on the timescale of these ${ }^{19} \mathrm{~F}$ experiments. The ${ }^{89} \mathrm{Y}-{ }^{19} \mathrm{~F}$ doublet indicates that the binding event is of $1: 1$ stoichiometry. Since significant coupling between the nuclei is observed, we can infer that there must be some orbital overlap between the Ln and $\mathrm{F}$ atoms and we would expect a contact contribution to the lanthanideinduced shift (LIS) of the fluoride (see below). Note that this does not necessarily imply covalent bonding since ${ }^{19} \mathrm{~F}$ nuclei are well known for showing coupling through non-bonding overlap of lone pairs. ${ }^{15}$

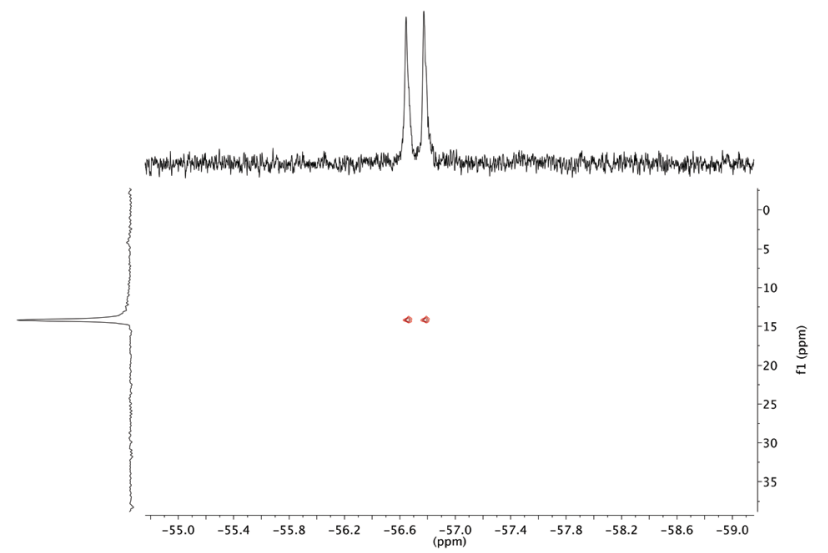

Fig. $2{ }^{19} \mathrm{~F}$ detected ${ }^{89} \mathrm{Y}-{ }^{19} \mathrm{~F}$ HMQC for a $\mathrm{D}_{2} \mathrm{O}$ solution of YDTMA and excess sodium fluoride with ${ }^{19} \mathrm{~F}$ on the horizontal axis and ${ }^{89} \mathrm{Y}$ on the vertical axis. The coupling in the vertical dimension is refocused during the evolution delay.

\section{NMR studies on paramagnetic complexes}

Proton NMR spectra of paramagnetic DTMA complexes with and without added fluoride were recorded in $\mathrm{D}_{2} \mathrm{O}$ for lanthanides from Pr to $\mathrm{Yb}$ across the series (excluding Pm and $\mathrm{Gd}$ ), and are shown in Fig. 5. Of the paramagnetic lanthanides, the NMR spectra of $\mathrm{Yb}$ complexes are most straightforward to interpret due to the domination of the pseudocontact contribution to the LIS felt by nearby nuclei, meaning that we can largely neglect the contact contribution and describe the shift using eqn (1). Furthermore, YbDTMA adopts only one conformation in solution, the square-antiprism (SAP). Upon addition of fluoride to a solution of YbDTMA, the original set of seven peaks appears to diminish in intensity and a new set of peaks grows in (Fig. 3). Given our other observations, we can surmise that these new peaks correspond to a fluoride-bound complex in slow exchange with the original hydrated species on this timescale. Notably, the range of chemical shifts spanned by the resonances of the new species is much smaller than that of the original (approximately 50 compared with $160 \mathrm{ppm}$ ). A two-dimensional exchange correlation spectrum (EXSY) confirms that the original peaks and the new set are in exchange (Fig. 3). Further inspection of the EXSY cross-peaks reveals that, on binding fluoride, the sign of the chemical shift of each proton changes, and the ordering of the peaks (from low frequency to high) is reversed. From eqn (1), it is clear that the only variable that can cause such changes is $B_{0}^{2}$. This NMR data suggests that the magnitude of $B_{0}^{2}$ is decreased and that its sign is reversed upon binding of fluoride in the axial site in place of water. The decreased magnitude of $B_{0}^{2}$ corroborates the observed changes in the EuDTMA emission spectrum.

Assignment of the resonances of YbDTMA, shown in Fig. 3, was achieved by comparison with similar compounds in the literature $^{16}$ and is confirmed by a linear plot of the observed LIS vs. $\left(3 \cos ^{2} \vartheta-1\right) / r^{3}$ according to eqn (1) for the six $\mathrm{CH}_{2}$ protons. Values of $\left(3 \cos ^{2} \vartheta-1\right) / r^{3}$ were taken from the closest available crystal structure $\left(\left(\right.\right.$ DyDTMA $\left.\left.\cdot \mathrm{H}_{2} \mathrm{O}\right)\left(\mathrm{PF}_{6}\right)_{3}\right) \cdot{ }^{12}$ The LIS is 

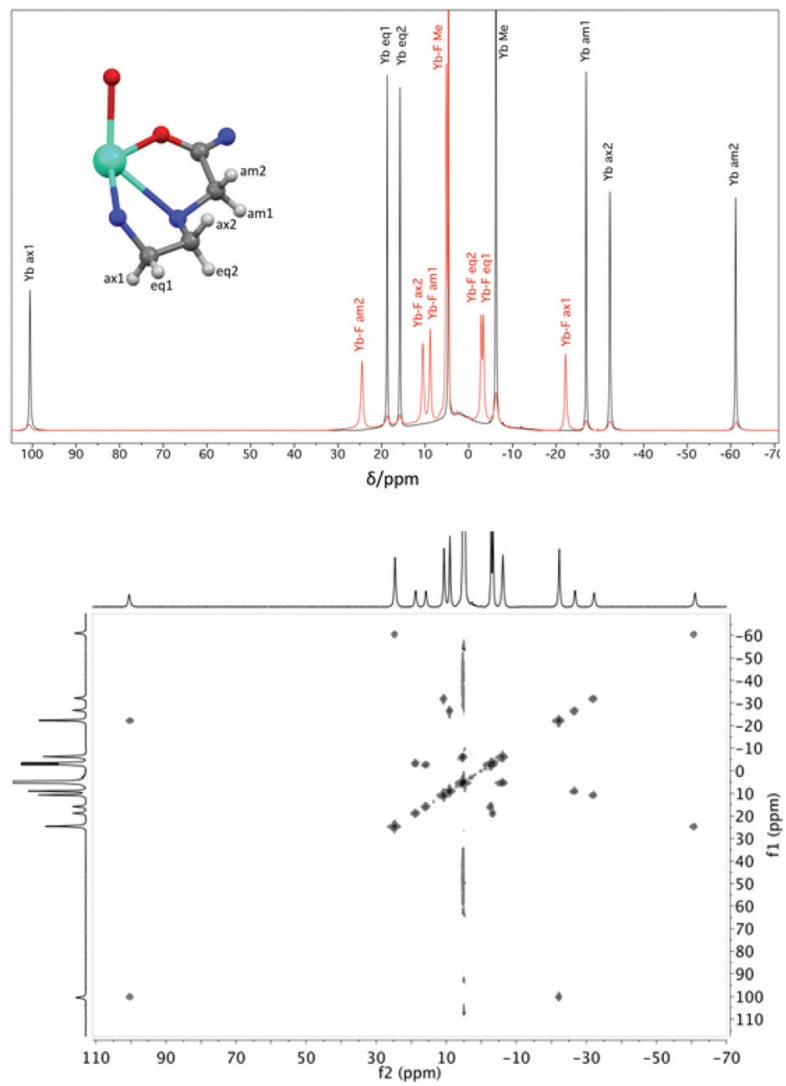

Fig. 3 Top: ${ }^{1} \mathrm{H}$ NMR spectra $\left(400 \mathrm{MHz}, 298 \mathrm{~K}, \mathrm{D}_{2} \mathrm{O}\right)$ of YbDTMA in the absence (black) and presence (red) of an excess of sodium fluoride, with assignments based on the labelled portion of the complex shown (using DyDTMA crystal structure ${ }^{12}$ ). Bottom: EXSY spectrum of YbDTMA with fluoride at $298 \mathrm{~K}$ with a mixing time of $1 \mathrm{~ms}$ at $500 \mathrm{MHz}$.

given by the observed shift ( $\left.\delta_{\text {obs }}\right)$ minus the diamagnetic contribution $\left(\delta_{\mathrm{D}}\right)$. Here, $2.9 \mathrm{ppm}$ was subtracted from each experimental shift as the estimated diamagnetic contribution, providing approximate values for the LIS. The six protons of the ligand backbone are labelled throughout this article, as axial (ax) $1 / 2$, equatorial (eq) $1 / 2$ and amide (am) $1 / 2$, according to the representation of the atoms shown in Fig. 3. The assignment of the protons of the fluoride-bound YbDTMA complex can be inferred from the EXSY spectrum. A plot of the LIS values vs. $\left(3 \cos ^{2} \vartheta-1\right) / r^{3}$ from the YbDTMA-F complex also gives a straight line, although Fig. 4 shows that the gradient of the line is now smaller and opposite in sign, consistent with the proposed changes to $B_{0}^{2}$. Analysis of the slopes of the Bleaney plots reveals that $B_{0}^{2}$ has been reduced to around $28 \%$ of its original value upon binding of fluoride.

Having assessed changes in the proton spectrum of YbDTMA with fluoride, we then investigated the other paramagnetic lanthanides. In each case, a second set of peaks emerged in slow exchange with the original peaks (Fig. 5). Analysis of the proton spectra of complexes of paramagnetic lanthanides other than $\mathrm{Yb}$ is complicated by (a) a significant contact shift contribution to the LIS, (b) large linewidths and

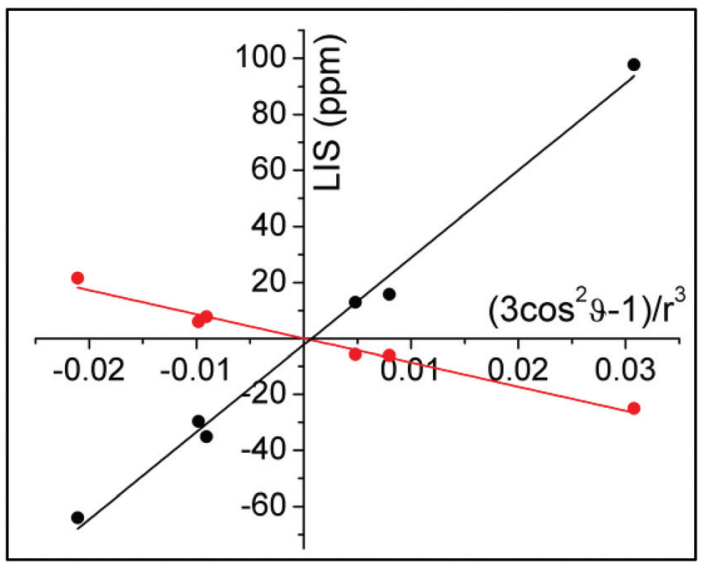

Fig. 4 Bleaney plots for YbDTMA proton resonances in the absence (black) and presence (red) of an excess of sodium fluoride in $\mathrm{D}_{2} \mathrm{O}$, using atomic coordinates from the crystal structure of DyDTMA. ${ }^{12}$

(c) the presence of multiple isomers in solution (SAP and TSAP). The presence of differing contact contributions to each proton shift in theory prevents the use of the LIS vs. $\left(3 \cos ^{2} \vartheta-\right.$ 1) $/ r^{3}$ relationship. Nevertheless, linear Bleaney plots are obtained for complexes with a relatively small contact contribution to their LIS (Tb, Dy, Ho, Er, Tm), by analogy with the YbDTMA assignment (see ESI $\dagger$ ). Whilst proton spectra of the DTMA complexes of the second half of the series show some resemblance to the YbDTMA spectrum, the contact shift distorts the spectrum of EuDTMA significantly and its Bleaney plot shows substantial deviation from linearity. It should be stressed that the contact shift contribution to the observed shifts of typical ligand protons cannot be neglected for the earlier lanthanides such as $\mathrm{Eu}^{3+}$. The assignment process is aided by inspection of $T_{1}$ relaxation times/linewidths considering the $r^{-6}$ dependence (where $r$ is the $\mathrm{Ln}-\mathrm{H}$ distance). Three protons have $r=3.6-3.7 \AA$, and the second set of three have $r=4.3 \AA$, resulting in two clusters of $T_{1}$ values. Assignment of the proton spectra of the fluoride-bound complexes is more difficult due to severe overlapping of peaks and the apparent unpredictability of the shifts, moreover EXSY spectra are largely uninformative due to the broad lines. However, reasonably linear Bleaney plots are achieved for $\mathrm{Tm}$, Er, Dy and $\mathrm{Tb}$ (see ESI $\dagger$ ). Data for HoDTMA could not be extracted since the greatly reduced chemical shift range results in severe peak overlap.

In order to assess changes to the pseudocontact and contact contributions to the ligand proton shifts upon fluoride binding, it is necessary to separate the two components. This is most commonly achieved using the so-called Reilley method. ${ }^{17}$ The paramagnetic influence on the shift is expressed as a sum of contact $\left(\delta_{\mathrm{C}}\right)$ and pseudocontact $\left(\delta_{\mathrm{P}}\right)$ contributions, that can be written as products of lanthanide dependant terms $\left(\left\langle S_{Z}\right\rangle\right.$ and $C_{J}$ respectively) and terms characteristic of the observed nuclei ( $F$ and $G$ respectively):

$$
\mathrm{LIS}=\delta_{\mathrm{obs}}-\delta_{\mathrm{D}}=\delta_{\mathrm{C}}+\delta_{\mathrm{P}}=\left\langle S_{Z}\right\rangle F+C_{J} G
$$



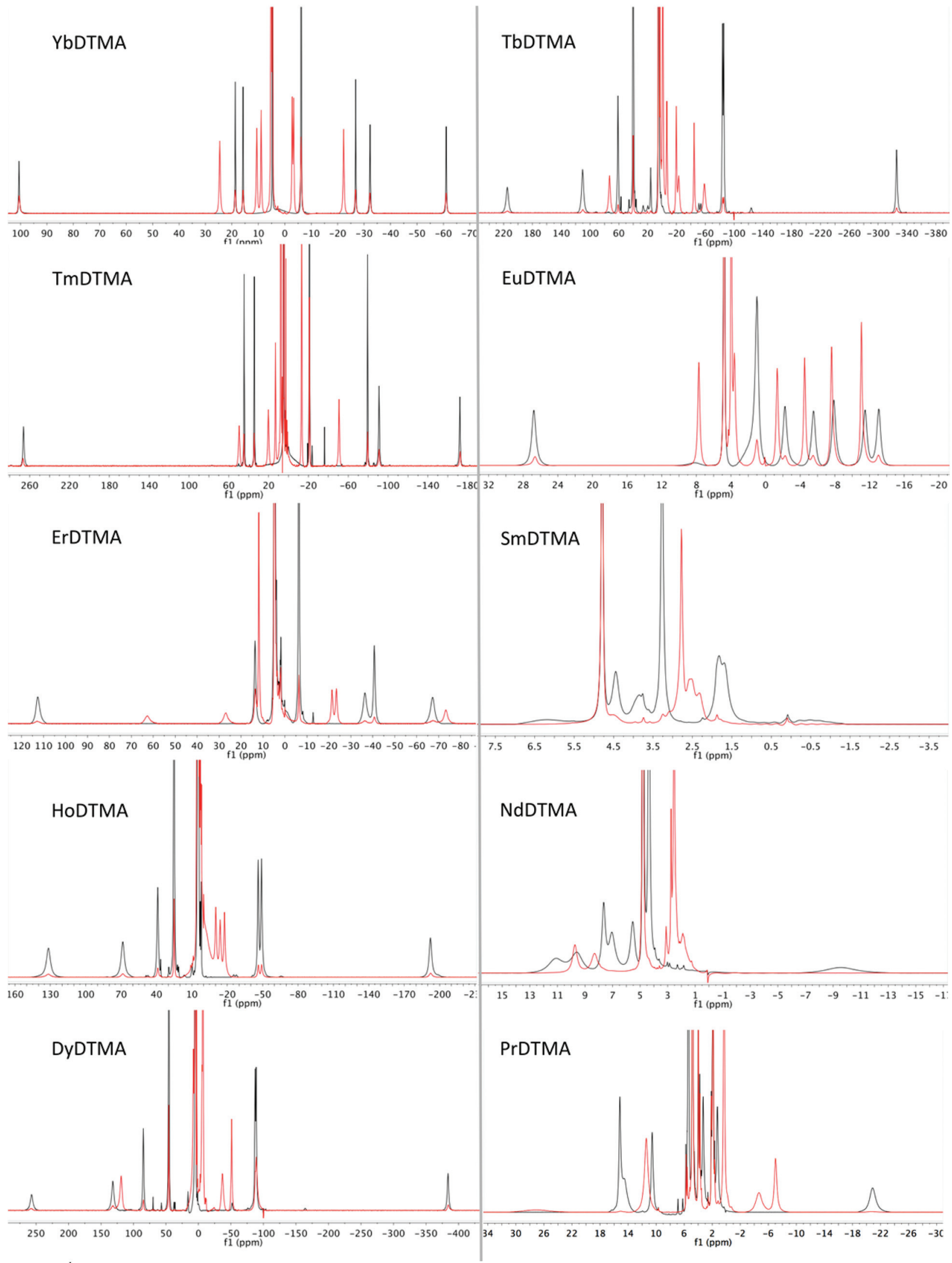

Fig. $5{ }^{1} \mathrm{H}$ NMR spectra of paramagnetic lanthanide complexes of DTMA from Pr to Yb (excluding Pm and Gd) in $\mathrm{D}_{2} \mathrm{O}$ at $298 \mathrm{~K}$ in the absence (black) and presence (red) of an excess of sodium fluoride. 
Theoretical values of $\left\langle S_{Z}\right\rangle$ and $C_{J}$ are tabulated in the literature. ${ }^{5,18,19}$ Eqn (2) can be written in two different linear forms:

Method A

$$
\frac{\mathrm{LIS}}{\left\langle S_{Z}\right\rangle}=\frac{C_{J}}{\left\langle S_{Z}\right\rangle} G+F
$$

Method B

$$
\frac{\mathrm{LIS}}{C_{J}}=\frac{\left\langle S_{Z}\right\rangle}{C_{J}} F+G
$$

Linear plots of LIS $/\left\langle S_{Z}\right\rangle$ vs. $C_{J} /\left\langle S_{Z}\right\rangle$ (Method A), and LIS $/ C_{J}$ vs. $\left\langle S_{Z}\right\rangle / C_{J}$ (Method B) across the series allow extraction of $F$ and $G$ values for each nucleus and thus the contact and pseudocontact contributions can be evaluated. Method A is more appropriate for pseudocontact dominated shifts and Method B for contact dominated shifts. The Reilley treatment makes a number of assumptions about the complexes across the series: (a) complexes are isostructural, (b) the crystal field parameter, $B_{0}^{2}$, is invariant, and (c) the hyperfine coupling constant, $A / \hbar$, is invariant.

Having assigned the proton resonances for a number of LnDTMA complexes, we were able to obtain Reilley plots (Method A) both in the presence and absence of fluoride with $R^{2}$ values above 0.9 (Fig. 6, Table 1). The methyl protons were also included in this treatment since they are easily identified. Values of $G$ (slope) and $F$ (intercept) extracted from the plots are given in Table 1 . The quality of the Reilley plots can also be judged by assessing the difference between the experimental shifts and those calculated based on the extracted $F$ and $G$ values for each proton; differences for YbDTMA for example are at most $5 \%$ (see ESI $\dagger$ ).

The $G$ values for each proton, which characterise the pseudocontact shifts, change sign with fluoride as expected and their magnitudes are reduced to between 21 and $31 \%$ of their original values, in agreement with the change observed from the Bleaney treatment for $\mathrm{Yb}$ above. Conversely, the changes in the $F$ values (which define the contact shifts) upon fluoride addition are not constant across all of the protons. Some protons, e.g. the equatorial protons, see only a small change in the contact contribution to their shifts. Meanwhile, for axial 1 and amide 2, $F$ changes in sign whilst their magnitudes vary little, meaning that the effect of the contact shift on these resonances is reversed. It is notable that the protons which feel the largest change in contact shift with fluoride are those closest to 90 and $180^{\circ}$ with respect to the molecular axis, though account should be taken of the change in anisotropy, discussed below. In general, the reduction in magnitude of the pseudocontact shift with fluoride results in an increased proportion of contact contribution to the shift since the magnitude of $F$ is only so drastically reduced in the case of axial proton 2. It is this relative increase in contact contribution combined with the large variation in the change of the contact shift with fluoride that makes the proton shifts of the fluoridebound complex difficult to assign.

The ${ }^{19} \mathrm{~F}$ NMR spectra of paramagnetic LnDTMA complexes with added fluoride revealed peaks for bound fluoride with
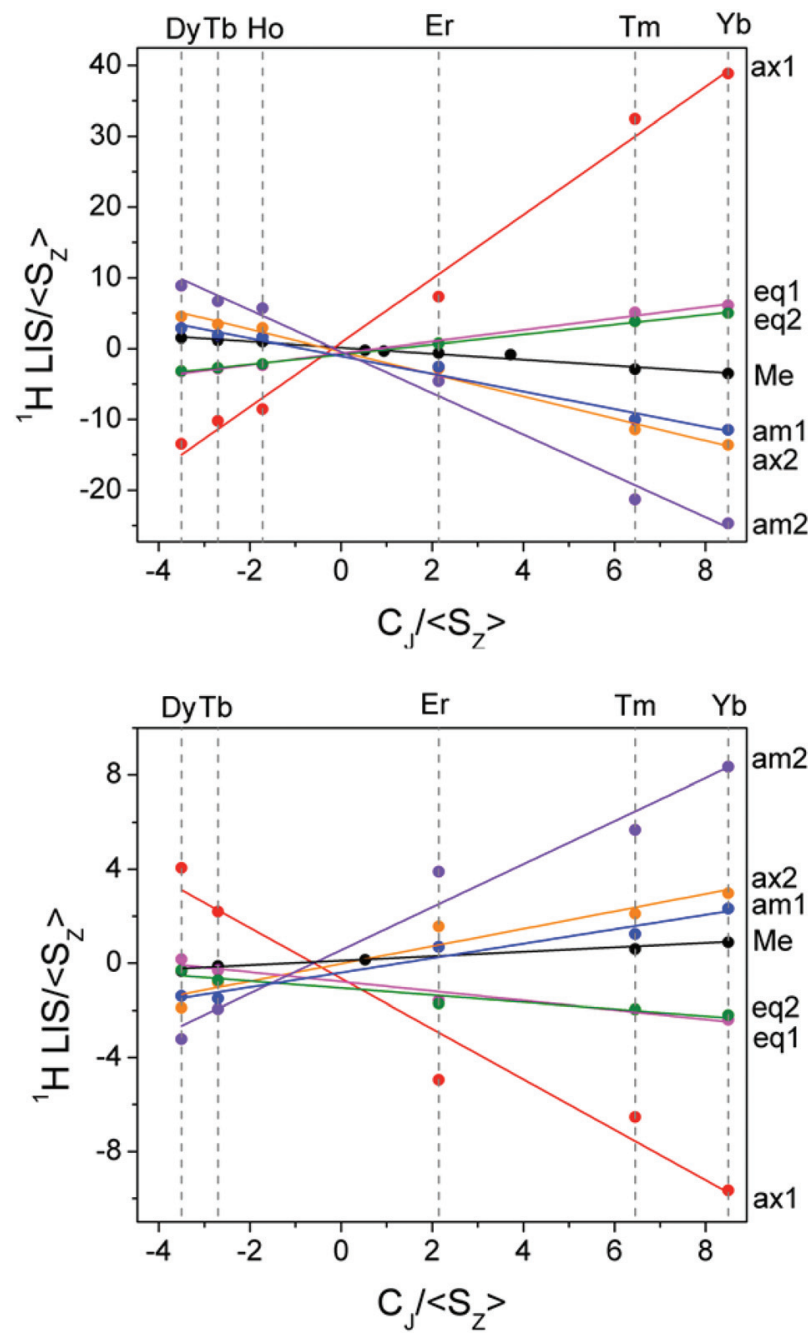

Fig. 6 Reilley plots (Method A) for the proton shifts of LnDTMA in $\mathrm{D}_{2} \mathrm{O}$ at $298 \mathrm{~K}$ without added fluoride (top) and for the fluoride-bound species (bottom).

Table 1 Values of $G$ and $F$ for ligand protons extracted from Reilley plots in the presence and absence of fluoride, with the $R^{2}$ value for each

\begin{tabular}{|c|c|c|c|c|c|c|}
\hline \multirow[b]{2}{*}{ Proton } & \multicolumn{2}{|c|}{$G$ (slope) } & \multicolumn{2}{|c|}{$F$ (intercept) } & \multicolumn{2}{|l|}{$R^{2}$} \\
\hline & No $F^{-}$ & $+\mathrm{F}^{-}$ & No $F^{-}$ & $+\mathrm{F}^{-}$ & No $F^{-}$ & $+\mathrm{F}^{-}$ \\
\hline Axial 1 & 4.52 & -1.07 & 0.82 & -0.64 & 0.99 & 0.94 \\
\hline Axial 2 & -1.57 & 0.37 & -0.45 & -0.02 & 0.99 & 0.91 \\
\hline Eq. 1 & 0.81 & -0.20 & -0.65 & -0.78 & 0.99 & 0.94 \\
\hline Eq. 2 & 0.70 & -0.15 & -0.83 & -1.05 & 1.00 & 0.91 \\
\hline Amide 1 & -1.25 & 0.31 & -1.05 & -0.40 & 0.98 & 0.95 \\
\hline Amide 2 & -2.93 & 0.92 & -0.42 & 0.55 & 0.99 & 0.96 \\
\hline Methyl & -0.42 & 0.09 & 0.14 & 0.05 & 0.97 & 0.98 \\
\hline
\end{tabular}
plot

large shifts, although only in a few cases: Nd $231 \mathrm{ppm}, \mathrm{Sm}$ -112 ppm, Eu -479 ppm, Tm -1600 ppm, Yb -858 ppm. These peaks display short $T_{1}$ relaxation times, as expected ( $\mu$ sms order). In each case, the $T_{1}$ of the free fluoride peak at 
-122 ppm is diminished compared to a fluoride solution containing no LnDTMA. The free fluoride $T_{1}$ becomes longer as the concentration of fluoride is increased, consistent with a weighted average value due to exchange. Saturation of the bound fluoride peak results in diminished intensity of the free fluoride peak and vice versa, confirming that chemical exchange between the two is taking place. The ratio of the intensity of the bound fluoride peak to that of the triflate counterion gives an estimation of the proportion of the complex that has a bound fluoride ion. The same ratio can be obtained from the proton spectra of the same sample by integration of the two sets of peaks, and this ratio matched well with that from the ${ }^{19} \mathrm{~F}$ spectra. This is a good indication that the shifted ${ }^{19} \mathrm{~F}$ peaks correspond to the same species as the proton signals that emerge on addition of fluoride. The linewidths of these bound fluoride peaks are generally a lot broader than the analogous peaks of the diamagnetic complexes, although that for $\mathrm{Eu}$ is less than twice as wide as for Lu. The observed lines are perhaps not as broad as might be anticipated, ${ }^{20}$ and the same observation was made recently for the ${ }^{17} \mathrm{O}$ resonances of LnDOTA complexes, ${ }^{21}$ although it is likely that the analogous peaks for the remaining paramagnetic lanthanides are undetectable due to extensive broadening. Line broadening of ${ }^{19} \mathrm{~F}^{-}$in this case is obviously affected by exchange as well as proximity to paramagnetic ions.

The contact and pseudocontact contributions to the bound fluoride shift can be assessed in the same way as for the protons. Reilley analysis with the available shifts gives good linear plots $\left(R^{2} \geq 0.93\right)$ for the metals available using both Methods A and B (see ESI $\dagger$ ), using the shift of bound fluoride in the $\mathrm{Lu}$ complex to subtract the diamagnetic contribution. Samarium is anomalous in both cases and is omitted from the analysis; $\mathrm{Sm}^{3+}$ is well known to have a highly variable $\left\langle S_{Z}\right\rangle$ and therefore be an outlier in such treatments. ${ }^{18}$ There is good agreement between the $F$ and $G$ values given by the two plots and average values of $F=-37.7$ and $G=-28.0$ are obtained. The much greater magnitudes of the $F$ and $G$ values compared with those of the ligand protons, despite similar gyromagnetic ratios, reflect the smaller values of $r$ and $\theta$, and the increased influence of the unpaired electron density on the ${ }^{19} \mathrm{~F}$ nucleus. As expected for a nucleus directly bound to the lanthanide centre, there is a significant contact contribution to the fluoride LIS.

These assessments of the proton and fluorine shift data indicate that the assumptions of the Reilley method hold true for LnDTMA complexes and their fluoride-adducts. In particular, our conclusions about changes to the sign and magnitude of the crystal field parameter $B_{0}^{2}$ can be applied across the lanthanide series.

\section{Exchange rates}

Some indications about the rate of exchange of fluoride at the lanthanide centre can be gleaned from NMR spectra. Firstly, the presence of the ${ }^{89} \mathrm{Y}-{ }^{19} \mathrm{~F}$ doublet suggests that fluoride is binding to the yttrium centre in a process that is not appreciably faster than $64 \mathrm{~Hz}$. Secondly, ${ }^{1} \mathrm{H}$ and ${ }^{19} \mathrm{~F}$ NMR indicate that the fluoride exchange process is faster for LuDTMA than YDTMA (see above). Since we would expect binding at the axial site to take place via a dissociative mechanism by analogy with water exchange, ${ }^{12,22}$ we can infer that the faster rate for Lu may be due to an increased rate of dissociation of the bound species due to steric crowding at the axial site.

Exchange rates can be quantified using NMR techniques. Selective inversion experiments, monitoring the intensity of the exchanging peaks after a selective pulse, are difficult to carry out on these systems due to large chemical shift separations and fast $T_{1} \mathrm{~s}$. When this technique was attempted with the ${ }^{19} \mathrm{~F}$ spectrum of YDTMA, we were unable to fit the data to a simple 2-site exchange. Since the fluoride-bound species can be formed by exchange with either water or fluoride in the axial site, distinct rates will become convoluted and analysis becomes difficult with the available data. We therefore attempted to obtain approximate, averaged rates using a saturation transfer technique with ${ }^{19} \mathrm{~F}$ spectra based on a previously described method (see ESI $\dagger$ for details). ${ }^{23}$ Rates for Eu, Y, Yb and LuDTMA complexes were calculated to be around $1 \mathrm{~s}^{-1}$, although this value is affected by concentration and $K$. The exchange of fluoride for water at the lanthanide can therefore be considered to be a relatively slow process in comparison with, for example, the exchange of water $\left(k_{\mathrm{ex}}=6.4( \pm 1.5) \times 10^{3}\right.$ $\mathrm{s}^{-1}$ for EuDTMA, SAP isomer). ${ }^{12}$

\section{Determining association constants with fluoride}

The association of fluoride with LnDTMA was quantified to give binding constants $(K)$ using NMR and luminescence techniques. In all cases a 1:1 binding model was used within the DYNAFIT program. ${ }^{24}$ Association constants with confidence intervals are given in Table 2.

We were able to use changes to the ${ }^{1} \mathrm{H}$ NMR spectra upon titration with sodium fluoride to assess fluoride binding for $\mathrm{Eu}^{3+}, \mathrm{Y}^{3+}, \mathrm{Yb}^{3+}$ and $\mathrm{Lu}^{3+}$ DTMA complexes. For the systems in slow exchange $\left(\mathrm{Eu}^{3+}, \mathrm{Y}^{3+}, \mathrm{Yb}^{3+}\right)$, the binding isotherms were obtained by integration of the signals relative to the watersoluble standard DSS (4,4-dimethyl-4-silapentane-1-sulfonic acid), while for LuDTMA the shifting resonances were moni-

Table 2 Association constants, $K$, for LnDTMA(OTf) 3 complexes with sodium fluoride in $\mathrm{D}_{2} \mathrm{O}$ at $298 \mathrm{~K}$. 95\% confidence intervals are given in square brackets ${ }^{a}$

\begin{tabular}{llll}
\hline & $K / \mathrm{M}^{-1}$ & & \\
\cline { 2 - 4 } & ${ }^{1} \mathrm{H} \mathrm{NMR}$ & ${ }^{19} \mathrm{~F} \mathrm{NMR}$ & Luminescence \\
\hline EuDTMA & $57.5[47.7-69.5]$ & $47.4[30.0-80.2]$ & $78.9[56.2-113.9]$ \\
YDTMA & $48.7[35.6-66.5]$ & $31.4[22.4-45.0]$ & - \\
YbDTMA & $9.38[7.64-11.43]$ & - & - \\
LuDTMA & $10.2[8.9-11.7]$ & $12.8[9.7-17.2]$ & -
\end{tabular}

${ }^{a}$ In all cases, the data was modelled according to a $1: 1$ binding model, using the equilibrium:

$\left(\text { LnDTMA }-\mathrm{H}_{2} \mathrm{O}\right)^{3+}+\mathrm{F}^{-} \rightleftharpoons(\text { LnDTMA }-\mathrm{F})^{2+}+\mathrm{H}_{2} \mathrm{O}$

$K=\frac{\left[(\text { LnDTMA }-\mathrm{F})^{2+}\right]}{\left[\left(\operatorname{LnDTMA}-\mathrm{H}_{2} \mathrm{O}\right)^{3+}\right]\left[\mathrm{F}^{-}\right]}$ 
tored. For titrations by ${ }^{19} \mathrm{~F}$ NMR, the increasing intensity of the bound fluoride peak was monitored with respect to the intensity of the $\mathrm{CF}_{3} \mathrm{SO}_{3}{ }^{-}$counterion. Changes to the europium emission spectrum provided a third method of monitoring the interaction. The ratios of the intensity of the hypersensitive $\Delta J=2$ transition $v s$. those of the $\Delta J=1$ and 4 transitions were used to monitor the changes in the local coordination sphere of the $\mathrm{Eu}^{3+}$ ion. For further details of all procedures, see ESI. $\dagger$

Where multiple independent titrations were carried out on the same complex by different methods, the values obtained are within error of one-another, confirming that the observed changes in the spectra correspond to the same event. However, there does seem to be a significant difference in $K$ across the series with $\mathrm{Eu}$ and $\mathrm{Y}$ complexes having higher association constants than $\mathrm{Yb}$ and $\mathrm{Lu}$ complexes. It might be expected that decreasing ionic radii across the series would infer increasing binding constants due to increasing charge density [9 coordinate ionic radii $\left.(\mathrm{pm}): \mathrm{Eu}^{3+} 112, \mathrm{Y}^{3+} 108, \mathrm{Yb}^{3+} 104, \mathrm{Lu}^{3+} 103\right] .{ }^{25}$ However, the decreased ionic size also results in the ligand enveloping more of the lanthanide ion and so less space is available in the axial site for ligand binding. The two factors are competing and in the case of fluoride, the steric factor appears to be dominant. A similar conclusion was postulated by Aime et al. on comparison of fluoride's interaction with $\mathrm{Yb}$ and Eu complexes of DOTA. ${ }^{9}$

It should be noted that the values obtained here are small in comparison to those recently reported by Charbonnière and co-workers,${ }^{10}$ though their system forms $2: 1$ complexes in which the fluoride binds between two lanthanide centres as a result of strong interactions between hydrophobic domains in both lanthanide complexes. In our case, no improvement in fit was observed with $2: 1$ binding models, suggesting that $1: 1$ complexation does indeed occur. Furthermore the observation of a doublet for fluoride bound to the yttrium complex bears out $1: 1$ complexation in our system. The binding constants are likely to reflect the thermodynamic costs of solvent reorganisation and desolvation of both the lanthanide centre and the fluoride ion, as well as the electrostatic attraction between the lanthanide and the fluoride.

\section{Summary and conclusions}

Binding of fluoride by lanthanide DTMA complexes results in dramatic changes in the behaviour of the fluoride bound species relative to that of their analogues with coordinated water, which result from a change in the sign and magnitude of the crystal field term. While we have discussed these changes in terms of a simple crystal field splitting parameter and related this directly to $B_{0}^{2}$, as is commonly done for axially symmetric complexes, it seems likely that these dramatic changes would also affect higher order terms in $B_{q}^{k}{ }^{7}$ We can interpret the observed phenomena in terms of a change in the anisotropy of the magnetic suceptibility tensor i.e. moving from a prolate system in which the anisotropic distribution of electrons aligns with the axis of symmetry to an oblate one, or
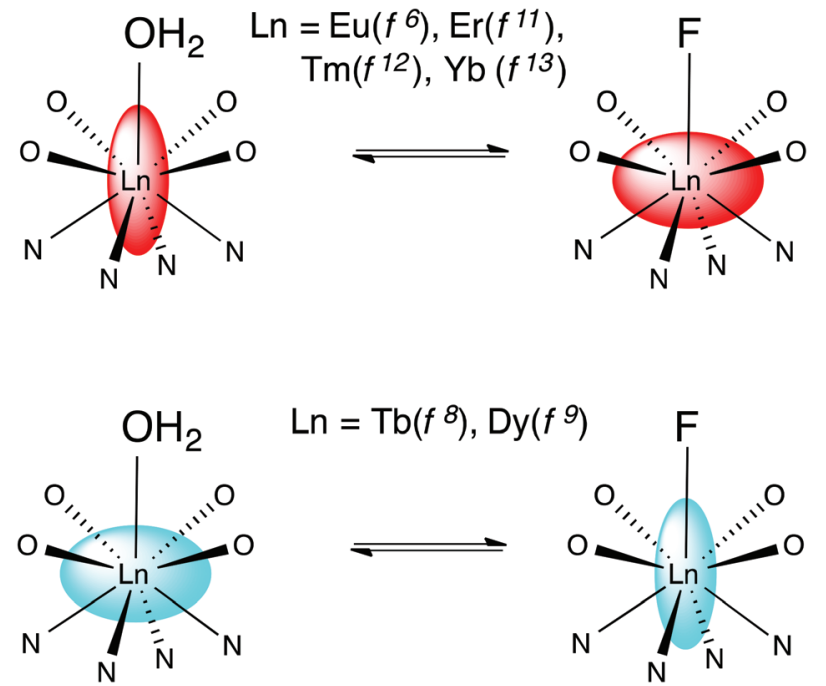

Scheme 2 Representing the change in magnetic anisotropy upon fluoride binding for the later lanthanides.

vice versa. Scheme 2 offers a pictorial representation of these changes for the later lanthanides.

It is clear from these results that the ligand donor set is of vital importance in determining the properties of lanthanide complexes. That importance has already become apparent in the field of lanthanide magnetism, and it clearly has relevance for a wide variety of areas from ligand design to MRI applications. Furthermore, calculation of ground states for lanthanide compounds must take account of ligand field if we are to explain anisotropy properly.

It is also worth noting that, despite binding weakly to the metal centre, slow exchange of fluoride implies significant reorganisation of the solvation sphere upon binding. We are exploring ways to exploit both this slow exchange and the dominance of the axial ligand in designing and applying new ligand systems.

\section{Acknowledgements}

The Authors acknowledge the Universities of Oxford and Durham for support. The research leading to these results has received funding from the European Research Council under the European Union's seventh Framework Programme (FP7/ 2007_2013)/ERC-Advanced Grant Agreement Number 267426.

\section{Notes and references}

1 (a) P. Caravan, J. J. Ellison, T. J. McMurry and R. B. Lauffer, Chem. Rev., 1999, 99, 2293-2352; (b) S. Faulkner and O. A. Blackburn, in The Chemistry of Molecular Imaging, ed. N. Long and W. T. Wong, Wiley, Chichester, 2015, ch. 8; (c) J.-C. G. Bünzli and C. Piguet, Chem. Soc. Rev., 2005, 34, 
1048-1077; (d) S. V. Eliseeva and J.-C. G. Bünzli, Chem. Soc. Rev., 2009, 39, 189-227.

2 (a) S. Viswanathan, Z. Kovacs, K. N. Green, S. J. Ratnakar and A. D. Sherry, Chem. Rev., 2010, 110, 2960-3018; (b) M. Woods, D. E. Woessner and A. D. Sherry, Chem. Soc. Rev., 2006, 35, 500-511; (c) P. Harvey, A. M. Blamire, J. I. Wilson, K.-L. N. A. Finney, A. M. Funk, P. K. Senanayake and D. Parker, Chem. Sci., 2013, 4, 42514258.

3 (a) D. N. Woodruff, R. E. P. Winpenny and R. A. Layfield, Chem. Rev., 2013, 113, 5110-5148; (b) L. Sorace, C. Benelli and D. Gatteschi, Chem. Soc. Rev., 2011, 40, 3092-3104; (c) J. D. Rinehart and J. R. Long, Chem. Sci., 2011, 2, 20782085.

4 (a) S. J. Butler and D. Parker, Chem. Soc. Rev., 2013, 42, 1652-1666; (b) C. P. Montgomery, B. S. Murray, E. J. New, R. Pal and D. Parker, Acc. Chem. Res., 2009, 42, 925-937; (c) L. M. P. Lima and R. Tripier, Curr. Inorg. Chem., 2011, 1, 36-60; (d) C. M. G. Dos Santos, A. J. Harte, S. J. Quinn and T. Gunnlaugsson, Coord. Chem. Rev., 2008, 252, 25122527.

5 (a) B. Bleaney, J. Magn. Reson., 1972, 8, 91-100; (b) B. Bleaney, C. M. Dobson, B. A. Levine, R. B. Martin, R. J. P. Williams and A. V. Xavier, J. Chem. Soc., Chem. Commun., 1972, 791b-7793.

6 R. S. Dickins, D. Parker, J. I. Bruce and D. J. Tozer, Dalton Trans., 2003, 1264-1271.

7 A. M. Funk, K.-L. N. A. Finney, P. Harvey, A. M. Kenwright, E. R. Neil, N. J. Rogers, P. Kanthi Senanayake and D. Parker, Chem. Sci., 2015, 6, 1655-1662.

8 A. M. Funk, P. H. Fries, P. Harvey, A. M. Kenwright and D. Parker, J. Phys. Chem. A, 2013, 117, 905-917.

9 S. Aime, M. Botta, M. Fasano, M. P. M. Marques, C. F. Geraldes, D. Pubanz and A. E. Merbach, Inorg. Chem., 1997, 36, 2059-2068.

10 (a) T. Liu, A. Nonat, M. Beyler, M. Regueiro-Figueroa, K. Nchimi Nono, O. Jeannin, F. Camerel, F. Debaene, S. Cianférani-Sanglier, R. Tripier, C. Platas-Iglesias and L. J. Charbonnière, Angew. Chem., Int. Ed., 2014, 53, 72597263; (b) L. M. P. Lima, A. Lecointre, J.-F. Morfin, A. de Blas, D. Visvikis, L. J. Charbonnière, C. Platas-Iglesias and R. Tripier, Inorg. Chem., 2011, 50, 12508-12521; (c) R. Tripier, C. Platas-Iglesias, A. Boos, J.-F. Morfin and
L. Charbonnière, Eur. J. Inorg. Chem., 2010, 2010, 27352745.

11 O. A. Blackburn, N. F. Chilton, K. Keller, W. K. Myers, E. J. L. McInnes, A. M. Kenwright, P. D. Beer, C. R. Timmel and S. Faulkner, Angew. Chem., Int. Ed., 2015, DOI: 10.1002/ anie.201503421.

12 S. Aime, A. Barge, J. I. Bruce, M. Botta, J. A. K. Howard, J. M. Moloney, D. Parker, A. S. de Sousa and M. Woods, J. Am. Chem. Soc., 1999, 121, 5762-5771.

13 (a) P. Hanninen and H. Härmä, Lanthanide Luminescence, Springer, Heidelberg, Springer Series on Fluorescence, 2011, vol. 7; (b) C. Görller-Walrand and K. Binnemans, Rationalization of crystal field parametrization, in Handbook on the Physics and Chemistry of Rare Earths, ed. K. A. Gschneidner Jr. and L. Eyring, North-Holland Publishing, Amsterdam, 1996, ch. 155, vol. 23, p. 121.

14 A. Beeby, I. M. Clarkson, R. S. Dickins, S. Faulkner, D. Parker, L. Royle, A. S. de Sousa, J. A. G. Williams and M. Woods, J. Chem. Soc., Perkin Trans. 2, 1999, 493-504.

15 F. B. Mallory, J. Am. Chem. Soc., 1973, 95, 7747-7752.

16 (a) R. S. Dickins, J. A. Howard, C. L. Maupin, J. M. Moloney, D. Parker, J. P. Riehl, G. Siligardi and J. A. G. Williams, Chemistry, 1999, 5, 1095-1105; (b) J. H. Forsberg, R. M. Delaney, Q. Zhao, G. Harakas and R. Chandran, Inorg. Chem., 1995, 34, 3705-3715.

17 (a) C. N. Reilley, B. Good and J. Desreux, Anal. Chem., 1975, 47, 2110-2116; (b) S. Rigault, C. Piguet and J.-C. G. Bünzli, J. Chem. Soc., Dalton Trans., 2000, 2045-2053; (c) S. Rigault, C. Piguet, G. Bernardinelli and G. Hopfgartner, J. Chem. Soc., Dalton Trans., 2000, 4587-4600.

18 A. A. Pinkerton, M. Rossier and S. Spiliadis, J. Magn. Reson., 1985, 64, 420.

19 R. Golding and P. Pyykkö, Mol. Phys., 1973, 26, 1389-1396. 20 J. Peters, J. Huskens and D. Raber, Prog. Nucl. Magn. Reson. Spectrosc., 1996, 28, 283-350.

21 L. Fusaro and M. Luhmer, Inorg. Chem., 2014, 53, 8717.

22 F. A. Dunand, S. Aime and A. E. Merbach, J. Am. Chem. Soc., 2000, 122, 1506-1512.

23 R. L. Jarek, R. J. Flesher and S. K. Shin, J. Chem. Educ., 1997, 74, 978-982.

24 P. Kuzmič, Anal. Biochem., 1996, 237, 260-273.

25 R. D. Shannon, Acta Crystallogr., Sect. A: Cryst. Phys., Diffr., Theor. Gen. Cryst., 1976, 32, 751-767. 